\title{
Reactivity of 1-methylisoquinoline synthesis of pyrazolyl triazoloisoquinoline and thiadiazolyl isoquinoline derivatives
}

\author{
Hamdi Mahmoud Hassaneen*, Huwaida Mahmmed Elsayd Hassaneen, \\ Yasmin Shafie Mohammed
}

Department of Chemistry, Faculty of Science, Cairo University, Cairo, Egypt; ${ }^{*}$ Corresponding Author: Hamdihass@gmail.com

Received 18 May 2011; revised 10 June 2011; accepted 16 June 2011.

\begin{abstract}
The reaction of 1-methylisoquinoline 1 with hydrazonoyl halides 2 in ethanol in the presence of chitosan under microwave irradiation affords triazoloisoquinoline 4. Product 4 reacts with dimethylformamide-dimethylacetal to give enaminones 7 which react with hydrazonoyl halides to give pyrazolyl triazoloisoquinoline 13. Also, 1-methylisoquinoline 1 reacts with arylisothiocyanate to give thioanilide 15 which reacts with hydrazonoyl halides to give the corresponding thiadiazolyl isoquinoline derivatives 20, 24.
\end{abstract}

Keywords: [1,2,4] Triazolo [3,4-a] Isoquinolines; Enaminones; Hydrazonoyl Halides; Cycloaddition Reactions; Chitosan; Thioanilides; [1,3,4]

Thiadiazolylisoquinoline Derivatives

\section{INTRODUCTION}

Within the class of fused isoquinoline with their cardiovascular [1], anti-inflammatory [2], and antidepressant activites [3], [1,2,4]triazolo [3,4-a]isoquinolines are of considerable pharmaceutical and agricultural interest [4-7]. Therefore, the synthesis of this ring system is an attractive goal. We have previously reported the syntheses of triazoloisoquinoline and fused isoquinoline compounds via reaction of 3,4-dihydro-6,7-dimethoxyisoquinoline derivatives with hydrazonoyl halides in chloroform in the presence of triethylamine or in pyridine as catalyst and solvent [8-12]. The aim of the present study is to introduce a new synthetic method by replacing triethylamine in chloroform by the ecologically more acceptable catalyst chitosan $[13,14]$ and under microwave irradiation to enhance reaction rates [15-19] for the synthesis of $[1,2,4]$ triazolo[ $[3,4-a]$ isoquinolines which were found to be useful precursors for the synthesis of new enaminones 7. The latter compounds 7 were used to prepare carbonylpyrazolyl triazoloisoquinoline derivatives 13. Also, we synthesis thiadiazolyl isoquinoline derivatives 20,24 via a reaction of thioanilide 15 with hydrazonoyl halides 16 .

\section{EXPERIMENTAL}

The melting points were determined on a Stuart melting point apparatus and are uncorrected. The IR spectra were recorded as $\mathrm{KBr}$ pellets using a FTIR unit Brukervector 22 spectrophotometer. The ${ }^{1} \mathrm{H}$ NMR and ${ }^{13} \mathrm{C}$ NMR spectra were recorded in $\mathrm{CDCl}_{3}$ and DMSO-d $\mathrm{d}_{6}$ as solvents at $300 \mathrm{MHz}$ on Varian Gemini NMR spectrometer using TMS as internal standard. Chemical shifts are reported in $\delta$ units (ppm). Mass spectra were measured on a Shimadzu GCMS-QP-1000 EX mass spectrometer at $70 \mathrm{eV}$. Microwave used was CEM Discover labmate $^{\mathrm{TM}}$ microwave apparatus $(300 \mathrm{~W}$ with $\mathrm{Chem}-$ Driver $^{\mathrm{TM}}$ software). The elemental analyses were performed at the Micro Analytical Center, Cairo University.

\subsection{Synthesis of 1-(1-Aryl-8,9-Dimethoxy- 10b-Methyl-1,5,6,10b-Tetrahydro [1,2,4] Triazolo [3,4-a]lsquinlin-3-yl) Ethanone-4a-c}

Chitosan $(0.1 \mathrm{~g})$ was added to a solution of hydrazonoyl chloride 2 (1 mmol) and 1-methyl-3,4-dihydro-6, 7-dimethoxyisoquinoline $1(0.28 \mathrm{~g}, 1 \mathrm{mmol})$ in absolute ethanol $(5 \mathrm{~mL})$ at room temperature. The reaction mixture was irradiated under constant pressure $\left(11.2 \mathrm{Bar}, 80^{\circ} \mathrm{C}\right)$ for $10 \mathrm{~min}$ at a power of $300 \mathrm{~W}$. The hot solution was filtered to remove chitosan. After cooling, dilute $\mathrm{HCl}$ was added till $\mathrm{pH}$ became acidic and the solid was collected and crystallized from suitable solvent. The compounds prepared 4a-c with their physical data are listed in Tables 1 and 2. 


\subsection{Synthesis of (E)-1-(1-Aryl-8,9-Dimethoxy- 10b-Methyl-1,5,6,10b-Tetrahydro $[1,2,4]$ Triazolo-[3,4-a]lsoquinolin-3-yl)-3- Dimethylaminopropenone 7a-c}

A mixture of 1-(1-aryl-8,9-dimethoxy-10b-methyl-1, 5,6,10b-tetra-hydro[1,2,4]triazolo[3,4-a]isoquinolin-3-yl) ethanone $4(5 \mathrm{mmoles})$ and DMF-DMA $(3 \mathrm{~mL})$ was refluxed for $4 \mathrm{~h}$. The solid that precipitated was collected and crystallized from suitable solvent. The compounds prepared $7 \mathrm{a}-\mathrm{c}$ with their physical data are listed in Tables 1 and 2.

\subsection{Synthesis of Pyrazolyl Triazoloisoquinoline Derivatives 13a-i}

To a solution of the appropriate hydrazonoyl chloride 2 , $8,9(1 \mathrm{mmol})$ and enaminones $7(1 \mathrm{mmol})$ in absolute ethanol $(5 \mathrm{~mL})$ was added chitosan $(0.1 \mathrm{~g})$ at room temperature. The reaction mixture was irradiated under constant pressure $\left(11.2 \mathrm{Bar}, 80^{\circ} \mathrm{C}\right)$ for $10 \mathrm{~min}$ at a power of $300 \mathrm{~W}$. The hot solution was filtered to remove chitosan. After cooling, dilute $\mathrm{HCl}$ was added till $\mathrm{pH}$ became acidic and the solid was collected and crystallized from suitable solvent. The compounds prepared 13a-i with their physical data are listed in Tables $\mathbf{1}$ and $\mathbf{2}$.

\subsection{Synthesis of Thiadiazolyl Isoquinoline Derivatives 20a-j and 24a-g}

Equimolar quantities of thioanilides 15 and the appropriate hydrazonoyl halides were dissolved in absolute ethanol $(5 \mathrm{~mL})$ in the presence of chitosan $(0.1 \mathrm{~g})$ at room temperature. The reaction mixture was irradiated under constant pressure $\left(11.2 \mathrm{Bar}, 80^{\circ} \mathrm{C}\right)$ for $10 \mathrm{~min}$ at a power of $300 \mathrm{~W}$. The hot solution was filtered to remove chitosan. After cooling, dilute $\mathrm{HCl}$ was added till $\mathrm{pH}$ became acidic and the solid was collected and crystallized from suitable solvent to give the corresponding 1 , 3,4 -thiadiazoles. The compounds prepared 20a-j and 24a-g with their physical data are listed in Tables 1 and 2.

\section{RESULTS AND DISCUSSION}

The reaction of hydrazonoyl halides 2 with 1-methyl3, 4-dihydro-6, 7-dimethoxyisoquinoline 1 which has active group at $\mathrm{C}-1$ was studied.

The capacity of this dipolarophile 1 to behave as cyclic ketimine $1 \mathrm{~A}$ or as a secondary enamine $1 \mathrm{~B}$ has been discussed by many investigators $[20,21]$. Our aim point of interest whether addition of nitrilimines 3 occurred on the $\mathrm{C}=\mathrm{N}$ double bond of ketimine structure $1 \mathrm{~A}$ or enamine double bond of $1 \mathrm{~B}$ (Figure 1). Thus, reaction of 1 with nitrilimines 3, generated in situ by treatment of hydrazonoyl chlorides 2 with chitosan [22] in ethanol under microwave irradiation, gave products whose elemental analyses were compatible with triazole derivatives 4 , spyropyrazolines 5 or triazine derivatives 6 . The structures 5 and 6 were discarded on the basis of ${ }^{1} \mathrm{H}$ NMR evidences. For example, structure 5 will reveal two singlet signals assignable to $\mathrm{CH}_{2}$ and $\mathrm{NH}$ protons, while the other isomeric structure 6 is expected to reveal doublet and triplet assignable to $\mathrm{C} 1-\mathrm{CH}_{2}$ and $\mathrm{C} 11 \mathrm{~b}-\mathrm{CH}$ protons. Such signals were absent in the ${ }^{1} \mathrm{H}$ NMR spectra of the isolated products from reaction of 2 with 1 . Instead of these signals, the ${ }^{1} \mathrm{H}$ NMR spectra of the latter products showed one singlet signal at $\delta 2.08 \mathrm{ppm}$. The presence of such signal is compatible with the assigned structure 4. Indeed the proton resonance of the moiety $-\mathrm{N}=\mathrm{C}\left(\mathrm{CH}_{3}\right)$ - appears in the ${ }^{1} \mathrm{H}$ NMR spectra of the dipolarophile 1 at $\delta 2.3 \mathrm{ppm}$ [23]. This resonance was shifted to higher field in the ${ }^{1} \mathrm{H}$ NMR spectra (2.08 ppm) of the cycloadducts 4 indicating the conversion of such moiety to the saturated moiety $-\mathrm{N}-\mathrm{C}\left(\mathrm{CH}_{3}\right)$ - due to cycloaddition. Based on these findings the products isolated from the reaction mixture were assigned structure 4 (Figure 1).

Refluxing of 4a with DMFDMA for $4 \mathrm{~h}$ afforded a compound 7a which analyzed correctly for $\mathrm{C}_{24} \mathrm{H}_{28} \mathrm{~N}_{4} \mathrm{O}_{3}$ (Figure 2). Similarly compounds $4 \mathrm{~b}$, c were also pre0 pared by reaction of the corresponding 3-acetylisoquinoline derivatives with DMFDMA. The structures of the products 7 were fully established on the basis of spectral (MS, IR, ${ }^{1} \mathrm{H}$ NMR and ${ }^{13} \mathrm{C}$ NMR) and elemental analyses. For example, the ${ }^{1} \mathrm{H}$ NMR spectrum of 7 a showed two singlet signals at $\delta 2.96$ and $3.72 \mathrm{ppm}$ characteristic for - $\mathrm{N}(\mathrm{Me})_{2}$ group, two doublet signals at $\delta$ about 5.75 and $7.64 \mathrm{ppm}$ with coupling constant $J=13 \mathrm{~Hz}$ assignable to the two olefinic protons. The value of coupling constant is compatible with the $E$-configuration [24] depicted in Figure 2. Also its ${ }^{13} \mathrm{C}$ NMR spectrum showed two signals at $\delta 37.31$ and $45.48 \mathrm{ppm}$ assignable to $-\mathrm{N}(\mathrm{Me})_{2}$ group [25], in addition to the signals of other carbon atoms.

Reaction of enaminones 7 with nitrilimines 10 , generated in situ by the action of chitosan on the corresponding $\alpha$-ketohydrazonoyl halides 2, 8, 9 in refluxed ethanol gave, in each case, one isolable product as evidenced by TLC analysis and ${ }^{1} \mathrm{H}$ NMR spectra of the crude reaction mixture (Figure 3).

All the isolated cycloadducts gave satisfactory elemental analyses and mass spectral data which were consistent with either one of the two isomeric structures 13 or 14. Structure 14 was ruled out on the basis of ${ }^{1} \mathrm{H}$ NMR spectra. For example, in the pyrazole ring system C (4) is the most electron rich carbon, thus, H (4) in is expected to appear at higher field at $\delta 6.31 \mathrm{ppm}$. On the 
Table 1. Characterization data of the synthesized compounds.

\begin{tabular}{|c|c|c|c|c|c|c|c|c|}
\hline \multirow{2}{*}{ Compd. no. } & \multirow{2}{*}{$\begin{array}{c}\mathrm{Mp}\left({ }^{\circ} \mathrm{C}\right) \\
\text { solvent }\end{array}$} & \multirow{2}{*}{$\begin{array}{l}\text { Yield (\%), } \\
\text { color }\end{array}$} & \multirow{2}{*}{ Mol. Formula } & \multicolumn{5}{|c|}{$\%$ Analyses calcd., found } \\
\hline & & & & $\mathrm{C}$ & $\mathrm{H}$ & $\mathrm{N}$ & $\mathrm{Cl}$ & $\mathrm{S}$ \\
\hline \multirow[t]{2}{*}{$4 \mathbf{a}$} & $\begin{array}{c}162 \\
\mathrm{EtOH}\end{array}$ & $\begin{array}{c}72 \\
\text { Yellow }\end{array}$ & \multirow{2}{*}{$\mathrm{C}_{21} \mathrm{H}_{23} \mathrm{~N}_{3} \mathrm{O}_{3}$} & $\begin{array}{l}69.02 \\
68.83\end{array}$ & 6.34 & 11.50 & & \\
\hline & & & & 68.83 & 0.13 & 11.01 & & \\
\hline \multirow{2}{*}{$4 b$} & 142 & 73 & \multirow{2}{*}{$\mathrm{C}_{22} \mathrm{H}_{25} \mathrm{~N}_{3} \mathrm{O}_{3}$} & 69.64 & 6.64 & 11.07 & & \\
\hline & $\mathrm{EtOH}$ & Yellow & & 69.45 & 6.58 & 11.25 & & \\
\hline \multirow{2}{*}{$4 c$} & 154 & 73 & \multirow{2}{*}{$\mathrm{C}_{21} \mathrm{H}_{22} \mathrm{ClN}_{3} \mathrm{O}_{3}$} & 63.08 & 5.55 & 10.51 & 8.87 & \\
\hline & $\mathrm{EtOH}$ & Yellow & & 62.93 & 5.75 & 10.66 & 8.63 & \\
\hline \multirow{2}{*}{$7 \mathbf{a}$} & 192 & 80 & \multirow{2}{*}{$\mathrm{C}_{24} \mathrm{H}_{28} \mathrm{~N}_{4} \mathrm{O}_{3}$} & 68.55 & 6.71 & 13.32 & & \\
\hline & $\mathrm{CH}_{3} \mathrm{CN}$ & Yellow & & 68.40 & 6.92 & 13.04 & & \\
\hline \multirow{2}{*}{$7 b$} & 186 & 79 & \multirow{2}{*}{$\mathrm{C}_{25} \mathrm{H}_{30} \mathrm{~N}_{4} \mathrm{O}_{3}$} & 69.10 & 6.96 & 12.89 & & \\
\hline & $\mathrm{CH}_{3} \mathrm{CN}$ & Yellow & & 68.87 & 7.03 & 13.06 & & \\
\hline \multirow{2}{*}{$7 c$} & 212 & 78 & \multirow{2}{*}{$\mathrm{C}_{24} \mathrm{H}_{27} \mathrm{ClN}_{4} \mathrm{O}_{3}$} & 63.36 & 5.98 & 12.31 & 7.79 & \\
\hline & DMF & Orange & & 63.11 & 6.01 & 12.02 & 7.53 & \\
\hline \multirow{2}{*}{$13 \mathbf{a}$} & 210 & 75 & \multirow{2}{*}{$\mathrm{C}_{31} \mathrm{H}_{29} \mathrm{~N}_{5} \mathrm{O}_{4}$} & 69.52 & 5.46 & 13.08 & & \\
\hline & $\mathrm{CH}_{3} \mathrm{CN}$ & Yellow & & 69.31 & 5.18 & 12.96 & & \\
\hline \multirow{2}{*}{$13 \mathrm{~b}$} & 170 & 78 & \multirow{2}{*}{$\mathrm{C}_{32} \mathrm{H}_{31} \mathrm{~N}_{5} \mathrm{O}_{4}$} & 69.93 & 5.69 & 12.74 & & \\
\hline & $\mathrm{EtOH}$ & Yellow & & 69.72 & 5.77 & 12.91 & & \\
\hline $13 c$ & 164 & 76 & & 65.32 & 4.95 & 12.29 & 6.22 & \\
\hline $15 \mathrm{c}$ & $\mathrm{EtOH}$ & Yellow & $\mathrm{C}_{31} \mathrm{H}_{28} \mathrm{C} \mathbb{1} \mathrm{N}_{5} \mathrm{U}_{4}$ & 65.05 & 5.11 & 12.38 & 6.16 & \\
\hline $13 d$ & 150 & 75 & & 67.95 & 5.52 & 12.38 & & \\
\hline 130 & $\mathrm{EtOH}$ & Yellow & $\mathrm{C}_{32} \mathrm{H}_{31} \mathrm{~N}_{5} \mathrm{U}_{5}$ & 67.68 & 5.46 & 12.41 & & \\
\hline $13 \mathrm{e}$ & 174 & 73 & $\mathrm{C}_{2} \mathrm{H}_{2} \mathrm{~N}_{8} \mathrm{O}_{5}$ & 68.38 & 5.74 & 12.08 & & \\
\hline $13 \mathrm{e}$ & $\mathrm{EtOH}$ & Yellow & $\mathrm{C}_{33} \mathrm{H}_{33} \mathrm{~N}_{5} \mathrm{U}_{5}$ & 68.15 & 5.94 & 11.93 & & \\
\hline $13 f$ & 158 & 76 & $\mathrm{C}_{2} \mathrm{H}_{2} \mathrm{ClN}_{5} \mathrm{O}_{5}$ & 64.05 & 5.04 & 11.67 & 5.91 & \\
\hline 151 & $\mathrm{EtOH}$ & Yellow & $\mathrm{C}_{32} \mathrm{H}_{30} \mathrm{CIN}_{5} \mathrm{U}_{5}$ & 63.87 & 5.31 & 11.83 & 6.12 & \\
\hline $13 \mathrm{~g}$ & 95 & 72 & $\mathrm{C}_{36} \mathrm{H}_{31} \mathrm{~N}_{5} \mathrm{O}_{4}$ & 72.35 & 5.23 & 11.72 & & \\
\hline & $\mathrm{EtOH}$ & Orange & $\mathrm{C}_{36} \mathrm{H}_{31} \mathrm{~N}_{5} \mathrm{U}_{4}$ & 72.51 & 5.42 & 11.47 & & \\
\hline $13 \mathrm{~h}$ & 180 & 73 & $\mathrm{C}_{37} \mathrm{H}_{33} \mathrm{~N}_{5} \mathrm{O}_{4}$ & 72.65 & 5.44 & 11.45 & & \\
\hline & $\mathrm{CH}_{3} \mathrm{CN}$ & Orange & & 72.54 & 5.41 & 11.26 & & \\
\hline $13 \mathbf{i}$ & 90 & 72 & & 68.40 & 4.78 & 11.08 & 5.61 & \\
\hline 101 & $\mathrm{EtOH}$ & Orange & $\mathrm{C}_{36} \mathrm{H}_{30} \mathrm{CIN}_{5} \mathrm{U}_{4}$ & 68.25 & 4.91 & 10.86 & 5.68 & \\
\hline $20 a$ & $174-175$ & 81 & $\mathrm{C}_{2} \mathrm{H}_{2} \mathrm{~N}=\mathrm{OS}$ & 63.85 & 5.58 & 9.31 & & 7.09 \\
\hline $20 \mathrm{a}$ & $\mathrm{CH}_{3} \mathrm{CN}$ & Yellow & $\mathrm{C}_{24} \mathrm{H}_{25} \mathrm{~N}_{3} \mathrm{U}_{4} \mathrm{~S}$ & 63.92 & 5.62 & 9.61 & & 6.92 \\
\hline $20 \mathrm{~b}$ & $208-210$ & 81 & $\mathrm{C}_{2} \mathrm{H}_{2} \mathrm{ClN}_{2} \mathrm{OSS}_{2}$ & 58.54 & 4.70 & 8.90 & 7.51 & 6.78 \\
\hline & DMF & Yellow & $\mathrm{C}_{23} \mathrm{H}_{22} \mathrm{ClN}_{3} \mathrm{U}_{4} \mathrm{~S}$ & 58.35 & 4.85 & 9.14 & 7.32 & 6.70 \\
\hline 20c & $201-202$ & 82 & $\mathrm{C}_{2} \mathrm{H}_{2} \mathrm{~N}_{3} \mathrm{O}_{4} \mathrm{~S}$ & 62.41 & 5.00 & 9.92 & & 7.56 \\
\hline $20 \mathrm{c}$ & DMF & Yellow & $\mathrm{C}_{22} \mathrm{H}_{21} \mathrm{~N}_{3} \mathrm{O}_{4} \mathrm{~S}$ & 62.53 & 4.89 & 9.68 & & 7.59 \\
\hline 20d & $210-211$ & 81 & $\mathrm{C}_{23} \mathrm{H}_{23} \mathrm{~N}_{3} \mathrm{O}_{4} \mathrm{~S}$ & 63.15 & 5.30 & 9.61 & & 7.32 \\
\hline & DMF & Yellow & 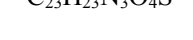 & 62.96 & 5.45 & 9.64 & & 7.49 \\
\hline $20 \mathrm{e}$ & $210-220$ & 79 & $\mathrm{C}_{2} \mathrm{H}_{2} \mathrm{ClN}_{2} \mathrm{O}_{4}$ & 57.71 & 4.40 & 9.18 & 7.74 & 6.99 \\
\hline 200 & DMF & Yellow & $\mathrm{C}_{22} \mathrm{H}_{20} \mathrm{C}-1 \mathrm{~N}_{3} \mathrm{O}_{4} \mathrm{~S}$ & 57.57 & 4.32 & 8.88 & 7.45 & 7.18 \\
\hline $20 \mathrm{f}$ & $266-268$ & 80 & & 64.19 & 4.56 & 11.52 & & 6.58 \\
\hline 201 & DMF & Yellow & $\mathrm{C}_{26} \mathrm{H}_{22} \mathrm{~N}_{4} \mathrm{O}_{4} \mathrm{~S}$ & 64.34 & 4.27 & 11.80 & & 6.74 \\
\hline $20 \boldsymbol{\sigma}$ & $279-280$ & 79 & $\mathrm{C}_{2} \mathrm{H}_{2} \mathrm{~N}_{2}$ & 64.79 & 4.83 & 11.19 & & 6.39 \\
\hline $20 \mathrm{~g}$ & DMF & Yellow & $C_{27} \cap_{24} \vee_{4} \mathrm{O}_{4} \mathrm{~S}$ & 64.99 & 5.07 & 10.88 & & 6.26 \\
\hline $20 \mathrm{~h}$ & $276-278$ & 82 & $\mathrm{C}_{2} \mathrm{H}_{21} \mathrm{ClN}_{4} \mathrm{O}_{4} \mathrm{~S}$ & 59.95 & 4.06 & 10.76 & 6.81 & 6.14 \\
\hline 2001 & DMF & Orange & $\mathrm{C}_{26} \mathrm{\Pi \Pi}_{21} \mathrm{CHN} \mathrm{N}_{4} \mathrm{U}_{4} \mathrm{~S}$ & 60.22 & 3.89 & 10.53 & 7.04 & 6.07 \\
\hline
\end{tabular}




\begin{tabular}{|c|c|c|c|c|c|c|c|c|}
\hline $20 \mathrm{i}$ & $\begin{array}{c}270-272 \\
\mathrm{DMF}\end{array}$ & $\begin{array}{c}80 \\
\text { Yellow }\end{array}$ & $\mathrm{C}_{24} \mathrm{H}_{20} \mathrm{~N}_{4} \mathrm{O}_{4} \mathrm{~S}_{2}$ & $\begin{array}{l}58.54 \\
58.58\end{array}$ & $\begin{array}{l}4.09 \\
4.25\end{array}$ & $\begin{array}{l}11.38 \\
11.17\end{array}$ & & $\begin{array}{l}13.00 \\
13.23\end{array}$ \\
\hline $20 \mathrm{j}$ & $\begin{array}{c}278-280 \\
\text { DMF }\end{array}$ & $\begin{array}{c}81 \\
\text { Yellow }\end{array}$ & $\mathrm{C}_{24} \mathrm{H}_{20} \mathrm{~N}_{4} \mathrm{O}_{5} \mathrm{~S}$ & $\begin{array}{l}60.50 \\
60.42\end{array}$ & $\begin{array}{l}4.23 \\
3.98\end{array}$ & $\begin{array}{l}11.76 \\
11.63\end{array}$ & & $\begin{array}{l}6.72 \\
6.81\end{array}$ \\
\hline $24 a$ & $\begin{array}{c}240-242 \\
\text { DMF }\end{array}$ & $\begin{array}{c}81 \\
\text { Yellow }\end{array}$ & $\mathrm{C}_{23} \mathrm{H}_{23} \mathrm{~N}_{3} \mathrm{O}_{3} \mathrm{~S}$ & $\begin{array}{l}65.55 \\
65.32\end{array}$ & $\begin{array}{l}5.50 \\
5.57\end{array}$ & $\begin{array}{c}9.97 \\
10.21\end{array}$ & & $\begin{array}{l}7.59 \\
7.74\end{array}$ \\
\hline $24 b$ & $\begin{array}{c}228-230 \\
\text { DMF }\end{array}$ & $\begin{array}{c}78 \\
\text { Orange }\end{array}$ & $\mathrm{C}_{22} \mathrm{H}_{20} \mathrm{ClN}_{3} \mathrm{O}_{3} \mathrm{~S}$ & $\begin{array}{l}59.80 \\
60.01\end{array}$ & $\begin{array}{l}4.56 \\
4.63\end{array}$ & $\begin{array}{l}9.51 \\
9.59\end{array}$ & $\begin{array}{l}8.02 \\
7.96\end{array}$ & $\begin{array}{l}7.24 \\
7.01\end{array}$ \\
\hline $24 c$ & $\begin{array}{l}200-201 \\
\text { DMF }\end{array}$ & $\begin{array}{c}84 \\
\text { Brown }\end{array}$ & $\mathrm{C}_{28} \mathrm{H}_{25} \mathrm{~N}_{3} \mathrm{O}_{3} \mathrm{~S}$ & $\begin{array}{l}69.55 \\
69.64\end{array}$ & $\begin{array}{l}5.21 \\
4.93\end{array}$ & $\begin{array}{l}8.69 \\
8.44\end{array}$ & & $\begin{array}{l}6.62 \\
6.68\end{array}$ \\
\hline $24 d$ & $\begin{array}{c}179-181 \\
\text { DMF }\end{array}$ & $\begin{array}{c}82 \\
\text { Red }\end{array}$ & $\mathrm{C}_{27} \mathrm{H}_{22} \mathrm{ClN}_{3} \mathrm{O}_{3} \mathrm{~S}$ & $\begin{array}{l}64.35 \\
64.42\end{array}$ & $\begin{array}{l}4.40 \\
4.72\end{array}$ & $\begin{array}{l}8.34 \\
8.48\end{array}$ & $\begin{array}{l}7.04 \\
6.83\end{array}$ & $\begin{array}{l}6.35 \\
6.57\end{array}$ \\
\hline $24 \mathrm{e}$ & $\begin{array}{c}210-211 \\
\text { DMF }\end{array}$ & $\begin{array}{c}79 \\
\text { Yellow }\end{array}$ & $\mathrm{C}_{27} \mathrm{H}_{24} \mathrm{~N}_{4} \mathrm{O}_{3} \mathrm{~S}$ & $\begin{array}{l}66.93 \\
67.11\end{array}$ & $\begin{array}{l}4.99 \\
5.07\end{array}$ & $\begin{array}{l}11.56 \\
11.31\end{array}$ & & $\begin{array}{l}6.60 \\
6.53\end{array}$ \\
\hline $24 f$ & $\begin{array}{c}170-172 \\
\text { DMF }\end{array}$ & $\begin{array}{c}80 \\
\text { Yellow }\end{array}$ & $\mathrm{C}_{28} \mathrm{H}_{26} \mathrm{~N}_{4} \mathrm{O}_{3} \mathrm{~S}$ & $\begin{array}{l}67.46 \\
67.26\end{array}$ & $\begin{array}{l}5.26 \\
4.95\end{array}$ & $\begin{array}{l}11.24 \\
11.13\end{array}$ & & $\begin{array}{l}6.42 \\
6.51\end{array}$ \\
\hline $24 \mathrm{~g}$ & $\begin{array}{c}219-220 \\
\mathrm{CH} 3 \mathrm{CN}\end{array}$ & $\begin{array}{c}83 \\
\text { Yellow }\end{array}$ & $\mathrm{C}_{27} \mathrm{H}_{23} \mathrm{C}_{1} \mathrm{~N}_{4} \mathrm{O}_{3} \mathrm{~S}$ & $\begin{array}{l}62.49 \\
62.57\end{array}$ & $\begin{array}{l}4.47 \\
4.15\end{array}$ & $\begin{array}{l}10.80 \\
10.88\end{array}$ & $\begin{array}{l}6.84 \\
6.62\end{array}$ & $\begin{array}{l}6.17 \\
5.93\end{array}$ \\
\hline
\end{tabular}

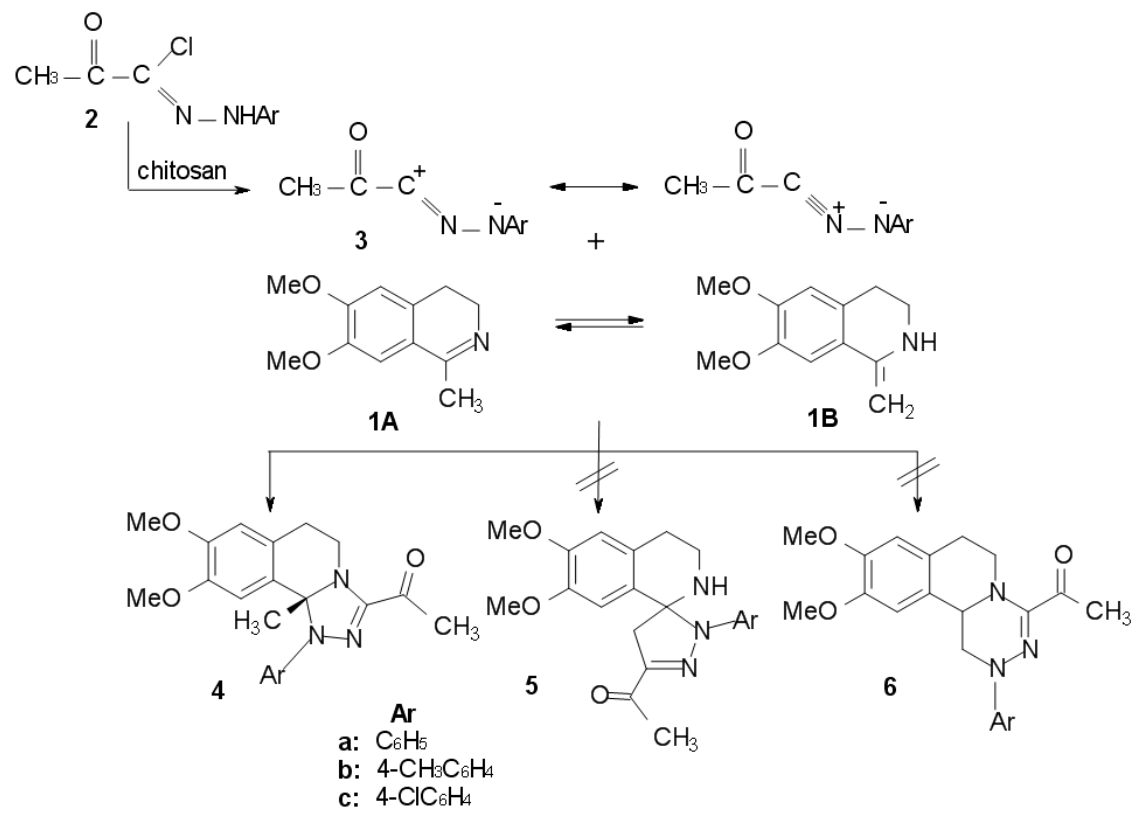

Figure 1. Synthesis of 3-acetyl-10b-methyl $[1,2,4]$ triazolo $[3,4-a]$ isoquinolines 4 .

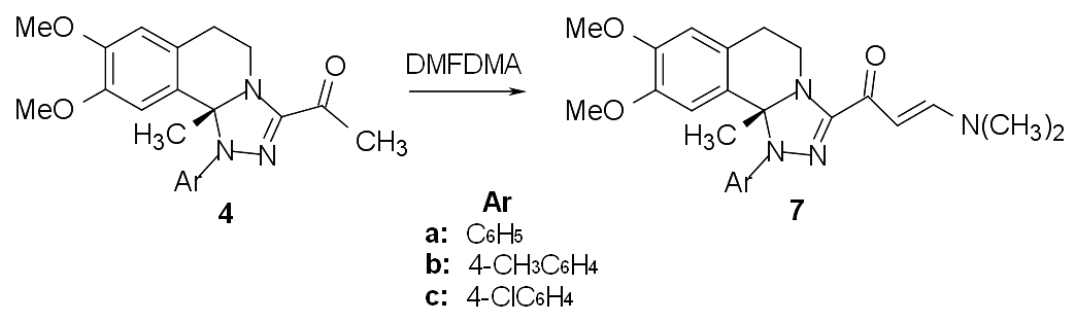

Figure 2. Synthesis of enaminones 7. 
Table 2. Spectra of the synthesized compounds.

\begin{tabular}{|c|c|}
\hline Compd. no. & Spectral data (IR, ${ }^{1} \mathrm{H}$ NMR, ${ }^{13} \mathrm{C}$ NMR and MS) \\
\hline $4 a$ & $\begin{array}{l}\mathrm{IR}(\mathrm{KBr}) v 1670(\mathrm{C}=\mathrm{O}) \mathrm{cm}^{-1} ;{ }^{1} \mathrm{H} \mathrm{NMR}\left(\mathrm{CDCl}_{3}\right) \delta 2.08(\mathrm{~s}, 3 \mathrm{H}), 2.37(\mathrm{~m}, 1 \mathrm{H}), 2.38(\mathrm{~s}, 3 \mathrm{H}), 2.80(\mathrm{~m}, 1 \mathrm{H}), 3.18(\mathrm{~s}, 3 \mathrm{H}), 3.2(\mathrm{~m}, \\
1 \mathrm{H}), 3.73(\mathrm{~s}, 3 \mathrm{H}), 4.60(\mathrm{~m}, 1 \mathrm{H}), 5.85(\mathrm{~s}, 1 \mathrm{H}), 6.40(\mathrm{~s}, 1 \mathrm{H}), 7.13-7.28(\mathrm{~m}, 5 \mathrm{H}) \mathrm{ppm} ;{ }^{13} \mathrm{C} \mathrm{NMR}\left(\mathrm{CDCl}_{3}\right) \delta 26.21,27.32,29.24, \\
40.22,54.9,55.35,90.09,109.20,111.04,123.94,125.09,127.16,127.69,128.70,143.19,146.04,147.75,147.97,189.88 \mathrm{ppm} \text {; } \\
\mathrm{MS}, m / z(\%): 365\left(\mathrm{M}^{+}, 10.9\right), 334(100.0), 103(22.0), 90(38.4), 76(30.8) .\end{array}$ \\
\hline $4 b$ & $\begin{array}{l}\mathrm{IR}(\mathrm{KBr}) v 1662(\mathrm{C}=\mathrm{O}) \mathrm{cm}^{-1} ;{ }^{1} \mathrm{H} \text { NMR }\left(\mathrm{CDCl}_{3}\right) \delta 2.05(\mathrm{~s}, 3 \mathrm{H}), 2.29(\mathrm{~s}, 3 \mathrm{H}), 2.34(\mathrm{~m}, 1 \mathrm{H}), 2.38(\mathrm{~s}, 3 \mathrm{H}), 2.84(\mathrm{~m}, 1 \mathrm{H}), 3.14(\mathrm{~m}, \\
1 \mathrm{H}), 3.23(\mathrm{~s}, 3 \mathrm{H}), 3.75(\mathrm{~s}, 3 \mathrm{H}), 4.65(\mathrm{~m}, 1 \mathrm{H}), 5.79(\mathrm{~s}, 1 \mathrm{H}), 6.41(\mathrm{~s}, 1 \mathrm{H}), 7.09-7.12(\mathrm{~m}, 4 \mathrm{H}) \mathrm{ppm} ;{ }^{13} \mathrm{C} \mathrm{NMR}\left(\mathrm{CDCl}_{3}\right) \delta 20.61, \\
26.24,27.40,29.20,40.35,54.76,55.40,90.21,109.45,111.02,124.39,127.11,127.86,129.28,135.16,140.60,145.96,147.68, \\
147.95,189.92 \mathrm{ppm} \text {; MS, } \mathrm{m} / \mathrm{z}(\%): 364\left(\mathrm{M}^{+}-15,100.0\right), 104(33.4), 90(20.2) .\end{array}$ \\
\hline $4 c$ & $\begin{array}{l}\mathrm{IR}(\mathrm{KBr}) v 1666(\mathrm{C}=\mathrm{O}) \mathrm{cm}^{-1} ;{ }^{1} \mathrm{H} \text { NMR }\left(\mathrm{CDCl}_{3}\right) \delta 2.03(\mathrm{~s}, 3 \mathrm{H}), 2.35(\mathrm{~m}, 1 \mathrm{H}), 2.39(\mathrm{~s}, 3 \mathrm{H}), 2.83(\mathrm{~m}, 1 \mathrm{H}), 3.13(\mathrm{~m}, 1 \mathrm{H}), 3.27(\mathrm{~s}, \\
3 \mathrm{H}), 3.74(\mathrm{~s}, 3 \mathrm{H}), 4.60(\mathrm{~m}, 1 \mathrm{H}), 5.86(\mathrm{~s}, 1 \mathrm{H}), 6.41(\mathrm{~s}, 1 \mathrm{H}), 7.14-7.25(\mathrm{~m}, 4 \mathrm{H}) \mathrm{ppm} ;{ }^{13} \mathrm{C} \text { NMR }\left(\mathrm{CDCl}_{3}\right) \delta 26.51,27.70,29.17 \text {, } \\
40.61,55.17,55.63,90.24,109.39,111.42,125.16,127.13,128.17,128.93,130.49,142.18,146.44,148.29,148.40,190.13 \mathrm{ppm} ; \\
\mathrm{MS}, \mathrm{m} / z(\%): 399\left(\mathrm{M}^{+}, 1.8\right), 384\left(\mathrm{M}^{+}-15,100.0\right), 90(8.6) .\end{array}$ \\
\hline
\end{tabular}

IR $(\mathrm{KBr}) \vee 1642(\mathrm{C}=\mathrm{O}) \mathrm{cm}^{-1} ;{ }^{1} \mathrm{H}$ NMR $\left(\mathrm{CDCl}_{3}\right) \delta 2.04(\mathrm{~s}, 3 \mathrm{H}), 2.38(\mathrm{~m}, 1 \mathrm{H}), 2.80(\mathrm{~s}, 3 \mathrm{H}), 2.88(\mathrm{~m}, 1 \mathrm{H}), 2.96(\mathrm{~s}, 3 \mathrm{H}), 3.16(\mathrm{~s}$, $3 \mathrm{H}), 3.19(\mathrm{~m}, 1 \mathrm{H}), 3.72(\mathrm{~s}, 3 \mathrm{H}), 4.80(\mathrm{~m}, 1 \mathrm{H}), 5.75(\mathrm{~d}, 1 \mathrm{H}), 5.89(\mathrm{~s}, 1 \mathrm{H}), 6.38(\mathrm{~s}, 1 \mathrm{H}), 7.04-7.24(\mathrm{~m}, 5 \mathrm{H}), 7.64(\mathrm{~d}, 1 \mathrm{H}) \mathrm{ppm} ;{ }^{13} \mathrm{C}$ 7a $\quad \mathrm{NMR}\left(\mathrm{CDCl}_{3}\right) \delta 27.85,29.10,37.31,40.42,45.48,54.95,55.37,88.63,93.13,109.52,110.93,123.80,124.29,128.02,128.04$, $128.50,144.36,145.88,147.67,150.13,152.23,179.19 \mathrm{ppm} ; \mathrm{MS}, \mathrm{m} / \mathrm{z}(\%): 420(\mathrm{M}+, 3.2), 405(\mathrm{M}+-15,87.9), 322(11.0), 98$ (100.0).

IR $(\mathrm{KBr}) \vee 1640(\mathrm{C}=\mathrm{O}) \mathrm{cm}^{-1} ;{ }^{1} \mathrm{H}$ NMR $\left(\mathrm{CDCl}_{3}\right) \delta 1.99(\mathrm{~s}, 3 \mathrm{H}), 2.26(\mathrm{~s}, 3 \mathrm{H}), 2.35(\mathrm{~m}, 1 \mathrm{H}), 2.80(\mathrm{~s}, 3 \mathrm{H}), 2.87(\mathrm{~m}, 1 \mathrm{H}), 2.95(\mathrm{~s}$, $3 \mathrm{H}), 3.16(\mathrm{~m}, 1 \mathrm{H}), 3.20(\mathrm{~s}, 3 \mathrm{H}), 3.71(\mathrm{~s}, 3 \mathrm{H}), 4.80(\mathrm{~m}, 1 \mathrm{H}), 5.74(\mathrm{~d}, 1 \mathrm{H}), 5.81(\mathrm{~s}, 1 \mathrm{H}), 6.37(\mathrm{~s}, 1 \mathrm{H}), 7.01-7.10(\mathrm{~m}, 4 \mathrm{H}), 7.63(\mathrm{~d}$ 1H) $\mathrm{ppm} ;{ }^{13} \mathrm{C}$ NMR $\left(\mathrm{CDCl}_{3}\right) \delta 20.77,28.10,29.25,37.38,42.71,45.49,54.96,55.58,88.96,93.40,109.94,111.08,124.49$, $128.13,128.38,129.25,134.42,141.99,145.97,147.82,150.18,152.37,179.46$ ppm; MS, m/z (\%): 434 (M+, 2.0), 419 (M+-15, 46.7), $336(10.5), 98(100.0)$.

IR $(\mathrm{KBr}) \vee 1640(\mathrm{C}=\mathrm{O}) \mathrm{cm}^{-1} ;{ }_{1}^{1} \mathrm{H}$ NMR $\left(\mathrm{CDCl}_{3}\right) \delta 1.98(\mathrm{~s}, 3 \mathrm{H}), 2.37(\mathrm{~m}, 1 \mathrm{H}), 2.79(\mathrm{~s}, 3 \mathrm{H}), 2.87(\mathrm{~m}, 1 \mathrm{H}), 2.94(\mathrm{~s}, 3 \mathrm{H}), 3.15(\mathrm{~m}$, $1 \mathrm{H}), 3.28(\mathrm{~s}, 3 \mathrm{H}), 3.71(\mathrm{~s}, 3 \mathrm{H}), 4.67(\mathrm{~m}, 1 \mathrm{H}), 5.75(\mathrm{~d}, 1 \mathrm{H}), 5.90(\mathrm{~s}, 1 \mathrm{H}), 6.38(\mathrm{~s}, 1 \mathrm{H}), 7.13-7.19(\mathrm{~m}, 4 \mathrm{H}), 7.63(\mathrm{~d}, 1 \mathrm{H}) \mathrm{ppm} ;{ }^{13} \mathrm{C}$ NMR $\left(\mathrm{CDCl}_{3}\right) \delta 28.17,28.99,37.42,40.77,44.98,55.18,55.61,88.71,93.22,109.68,111.30,124.97,127.99,128.48,128.67$, $129.48,143.35,146.25,148.08,150.81,152.68,179.17 \mathrm{ppm} ; \mathrm{MS}, \mathrm{m} / \mathrm{z}(\%): 454(\mathrm{M}+, 1.9), 439$ (M+-15, 29.2), 285 (31.5), 98 (100.0).

IR $(\mathrm{KBr})$ v $1692(\mathrm{C}=\mathrm{O}), 1645(\mathrm{C}=\mathrm{O}) \mathrm{cm}^{-1} ;{ }^{1} \mathrm{H} \mathrm{NMR}\left(\mathrm{CDCl}_{3}\right) \delta 2.13(\mathrm{~s}, 3 \mathrm{H}), 2.52(\mathrm{~m}, 1 \mathrm{H}), 2.63(\mathrm{~s}, 3 \mathrm{H}), 3.24(\mathrm{~s}, 3 \mathrm{H}), 3.38(\mathrm{~m}$, $1 \mathrm{H}), 3.41(\mathrm{~m}, 1 \mathrm{H}), 3.81(\mathrm{~s}, 3 \mathrm{H}), 4.79(\mathrm{~m}, 1 \mathrm{H}), 5.87(\mathrm{~s}, 1 \mathrm{H}), 6.53(\mathrm{~s}, 1 \mathrm{H}), 7.15-7.68(\mathrm{~m}, 10 \mathrm{H}), 8.33(\mathrm{~s}, 1 \mathrm{H}) \mathrm{ppm} ;{ }^{13} \mathrm{C} \mathrm{NMR}$ $\left(\mathrm{CDCl}_{3}\right) \delta 27.93,28.12,29.65,40.92,55.23,55.64,90.27,109.58,111.46,119.85,122.74,124.95,125.70,127.27,127.88$, $128.77,128.91,129.54,131.01,138.98,143.35,146.17,148.24,149.04,151.56,178.11,193.99 \mathrm{ppm} ; \mathrm{MS}, \mathrm{m} / \mathrm{z}(\%): 535(\mathrm{M}+$, $1.8), 520(\mathrm{M}+-15,100.0), 303(21.1), 213(13.9), 77(33.8)$.

IR $(\mathrm{KBr}) \vee 1694(\mathrm{C}=\mathrm{O}), 1645(\mathrm{C}=\mathrm{O}) \mathrm{cm}^{-1} ;{ }^{1} \mathrm{H}$ NMR $\left(\mathrm{CDCl}_{3}\right) \delta 2.10(\mathrm{~s}, 3 \mathrm{H}), 2.31(\mathrm{~s}, 3 \mathrm{H}), 2.52(\mathrm{~m}, 1 \mathrm{H}), 2.63(\mathrm{~s}, 3 \mathrm{H}), 3.26(\mathrm{~s}, 3 \mathrm{H})$, $3.30(\mathrm{~m}, 1 \mathrm{H}), 3.34(\mathrm{~m}, 1 \mathrm{H}), 3.83(\mathrm{~s}, 3 \mathrm{H}), 4.80(\mathrm{~m}, 1 \mathrm{H}), 5.82(\mathrm{~s}, 1 \mathrm{H}), 6.54(\mathrm{~s}, 1 \mathrm{H}), 7.01-7.69(\mathrm{~m}, 9 \mathrm{H}), 8.34(\mathrm{~s}, 1 \mathrm{H}) \mathrm{ppm} ;{ }^{13} \mathrm{C}$ $\operatorname{NMR}\left(\mathrm{CDCl}_{3}\right) \delta 20.85,27.97,28.14,29.59,40.97,55.04,55.63,90.32,109.75,111.38,119.89,122.77,125.34,127.20,127.87$, $128.86,129.43,129.54,131.04,135.78,139.02,140.71,146.05,148.17,148.86,151.60,178.01,194.10 \mathrm{ppm} ; \mathrm{MS}, \mathrm{m} / \mathrm{z}(\%): 549$ $(\mathrm{M}+, 1.1), 534(\mathrm{M}+-15,100.0), 317(13.2), 213(23.9), 91(11.8)$.

$13 \mathrm{c}$

IR $(\mathrm{KBr}) \vee 1692(\mathrm{C}=\mathrm{O}), 1650(\mathrm{C}=\mathrm{O}) \mathrm{cm}^{-1} ;{ }^{1} \mathrm{H}$ NMR $\left(\mathrm{CDCl}_{3}\right) \delta 2.08(\mathrm{~s}, 3 \mathrm{H}), 2.52(\mathrm{~m}, 1 \mathrm{H}), 2.62(\mathrm{~s}, 3 \mathrm{H}), 3.31(\mathrm{~m}, 1 \mathrm{H}), 3.33(\mathrm{~m}$, $1 \mathrm{H}), 3.35(\mathrm{~s}, 3 \mathrm{H}), 3.82(\mathrm{~s}, 3 \mathrm{H}), 4.78(\mathrm{~m}, 1 \mathrm{H}), 5.90(\mathrm{~s}, 1 \mathrm{H}), 6.55(\mathrm{~s}, 1 \mathrm{H}), 7.06-7.69(\mathrm{~m}, 9 \mathrm{H}), 8.32(\mathrm{~s}, 1 \mathrm{H}) \mathrm{ppm} ; \mathrm{MS}, \mathrm{m} / \mathrm{z}(\%): 581$ $(\mathrm{M}+, 1.0), 557(17.1), 556(46.1), 555(45.9), 554(100.0), 337$ (15.0), $213(43.8), 98(13.6)$.

IR $(\mathrm{KBr}) \vee 1728(\mathrm{C}=\mathrm{O}), 1635(\mathrm{C}=\mathrm{O}) \mathrm{cm}^{-1} ;{ }^{1} \mathrm{H}$ NMR $\left(\mathrm{CDCl}_{3}\right) \delta 1.31(\mathrm{t}, 3 \mathrm{H}), 2.17(\mathrm{~s}, 3 \mathrm{H}), 2.45(\mathrm{~m}, 1 \mathrm{H}), 3.20(\mathrm{~m}, 1 \mathrm{H}), 3.24(\mathrm{~s}$, $3 \mathrm{H}), 3.30(\mathrm{~m}, 1 \mathrm{H}), 3.80(\mathrm{~s}, 3 \mathrm{H}), 4.36(\mathrm{q}, 2 \mathrm{H}), 4.81(\mathrm{~m}, 1 \mathrm{H}), 5.95(\mathrm{~s}, 1 \mathrm{H}), 6.50(\mathrm{~s}, 1 \mathrm{H}), 7.16-7.69(\mathrm{~m}, 10 \mathrm{H}), 8.44(\mathrm{~s}, 1 \mathrm{H}) \mathrm{ppm} ;{ }^{13} \mathrm{C}$ $\operatorname{NMR}\left(\mathrm{CDCl}_{3}\right) \delta 14.13,27.82,29.57,40.66,55.22,55.69,61.52,90.21,109.37,111.37,120.17,123.14,124.02,125.36,127.64$, $127.94,128.27,129.01,129.46,131.10,138.94,144.05,145.92,147.65,148.81,149.22,162.54,176.33 \mathrm{ppm} ; \mathrm{MS}, \mathrm{m} / \mathrm{z}(\%): 550$ (M+-15, 100.0), 474 (30.1), 103 (45.1), 76 (82.4), 56 (47.6).

IR $(\mathrm{KBr}) \vee 1720(\mathrm{C}=\mathrm{O}), 1639(\mathrm{C}=\mathrm{O}) \mathrm{cm}^{-1} ;{ }^{1} \mathrm{H}$ NMR $\left(\mathrm{CDCl}_{3}\right) \delta 1.32(\mathrm{t}, 3 \mathrm{H}), 2.12(\mathrm{~s}, 3 \mathrm{H}), 2.30(\mathrm{~s}, 3 \mathrm{H}), 2.49(\mathrm{~m}, 1 \mathrm{H}), 3.18(\mathrm{~m}$, $1 \mathrm{H}), 3.25(\mathrm{~s}, 3 \mathrm{H}), 3.13(\mathrm{~m}, 1 \mathrm{H}), 3.79(\mathrm{~s}, 3 \mathrm{H}), 4.36(\mathrm{q}, 2 \mathrm{H}), 4.77(\mathrm{~m}, 1 \mathrm{H}), 5.88(\mathrm{~s}, 1 \mathrm{H}), 6.48(\mathrm{~s}, 1 \mathrm{H}), 7.10-7.67(\mathrm{~m}, 9 \mathrm{H}), 8.43(\mathrm{~s}$, 3H) ppm; 
IR $(\mathrm{KBr}) \vee 1720(\mathrm{C}=\mathrm{O}), 1639(\mathrm{C}=\mathrm{O}) \mathrm{cm}^{-1} ;{ }^{1} \mathrm{H} \mathrm{NMR}\left(\mathrm{CDCl}_{3}\right) \delta 1.31(\mathrm{t}, 3 \mathrm{H}), 2.12(\mathrm{~s}, 3 \mathrm{H}), 2.46(\mathrm{~m}, 1 \mathrm{H}), 3.09(\mathrm{~m}, 1 \mathrm{H}), 3.18(\mathrm{~m}$, $1 \mathrm{H}), 3.35(\mathrm{~s}, 3 \mathrm{H}), 3.79(\mathrm{~s}, 3 \mathrm{H}), 4.39(\mathrm{q}, 2 \mathrm{H}), 4.72(\mathrm{~m}, 1 \mathrm{H}), 5.98(\mathrm{~s}, 1 \mathrm{H}), 6.51(\mathrm{~s}, 1 \mathrm{H}), 7.15$ - $7.71(\mathrm{~m}, 9 \mathrm{H}), 8.42(\mathrm{~s}, 1 \mathrm{H}) \mathrm{ppm}$; MS, $\mathrm{m} / \mathrm{z}(\%): 584$ (M+-15, 100.0), 148 (21.1), 127 (22.1), 103 (61.5), $76(67.1)$.

IR $(\mathrm{KBr}) \vee 1675(\mathrm{C}=\mathrm{O}), 1631(\mathrm{C}=\mathrm{O}) \mathrm{cm}^{-1} ;{ }^{1} \mathrm{H}$ NMR $\left(\mathrm{CDCl}_{3}\right) \delta 2.10(\mathrm{~s}, 3 \mathrm{H}), 2.44(\mathrm{~m}, 1 \mathrm{H}), 3.21(\mathrm{~s}, 3 \mathrm{H}), 3.24(\mathrm{~m}, 1 \mathrm{H}), 3.28(\mathrm{~m}$ $1 \mathrm{H}), 3.82(\mathrm{~s}, 3 \mathrm{H}), 4.77(\mathrm{~m}, 1 \mathrm{H}), 5.83(\mathrm{~s}, 1 \mathrm{H}), 6.51(\mathrm{~s}, 1 \mathrm{H}), 7.02-8.04(\mathrm{~m}, 15 \mathrm{H}), 8.61(\mathrm{~s}, 1 \mathrm{H}) \mathrm{ppm} ;{ }^{13} \mathrm{C} \mathrm{NMR}\left(\mathrm{CDCl}_{3}\right) \delta 27.75$, $29.40,40.52,55.06,55.59,89.90,109.26,111.36,119.79,123.67,124.39,125.29,127.28,127.72,128.19,128.33,128.73$, $129.43,130.15,130.86,133.13,136.51,138.88,143.03,146.07,148.12,148.54,152.01,176.64,189.11 \mathrm{ppm} ; \mathrm{MS}, \mathrm{m} / \mathrm{z}(\%): 582$ $(\mathrm{M}+-15,100.0), 104$ (81.6), 76 (94.3).

IR $(\mathrm{KBr})$ v $1678(\mathrm{C}=\mathrm{O}), 1624(\mathrm{C}=\mathrm{O}) \mathrm{cm}^{-1} ;{ }^{1} \mathrm{H}$ NMR $\left(\mathrm{CDCl}_{3}\right) \delta 2.07(\mathrm{~s}, 3 \mathrm{H}), 2.29(\mathrm{~s}, 3 \mathrm{H}), 2.44(\mathrm{~m}, 1 \mathrm{H}), 3.20(\mathrm{~m}, 1 \mathrm{H}), 3.23(\mathrm{~s}$, $3 \mathrm{H}), 3.28(\mathrm{~m}, 1 \mathrm{H}), 3.83(\mathrm{~s}, 3 \mathrm{H}), 4.78(\mathrm{~m}, 1 \mathrm{H}), 5.79(\mathrm{~s}, 1 \mathrm{H}), 6.51(\mathrm{~s}, 1 \mathrm{H}), 6.90-8.04(\mathrm{~m}, 14 \mathrm{H}), 8.62(\mathrm{~s}, 1 \mathrm{H}) \mathrm{ppm} ;{ }^{13} \mathrm{C} \mathrm{NMR}$ $\left(\mathrm{CDCl}_{3}\right) \delta 20.78,27.83,29.39,40.65,54.95,55.66,90.03,109.51,111.34,119.89,123.72,124.83,127.26,127.75,128.25$, $128.51,129.33,129.48,130.21,130.97,133.15,135.41,136.61,139.00,140.51,146.02,148.13,148.40,152.10,176.55,189.29$ ppm; MS, m/z (\%): 611 (M+, 1.1), 596 (M+-15, 100.0), 324 (49.7), 104 (21.7), 76 (25.7).

IR $(\mathrm{KBr}) \vee 1678(\mathrm{C}=\mathrm{O}), 1639(\mathrm{C}=\mathrm{O}) \mathrm{cm}^{-1} ;{ }^{1} \mathrm{H} \mathrm{NMR}\left(\mathrm{CDCl}_{3}\right) \delta 2.04(\mathrm{~s}, 3 \mathrm{H}), 2.50(\mathrm{~m}, 1 \mathrm{H}), 3.20(\mathrm{~m}, 1 \mathrm{H}), 3.30(\mathrm{~s}, 3 \mathrm{H}), 3.36(\mathrm{~m}$, $1 \mathrm{H}), 3.83(\mathrm{~s}, 3 \mathrm{H}), 4.79(\mathrm{~m}, 1 \mathrm{H}), 5.83(\mathrm{~s}, 1 \mathrm{H}), 6.53(\mathrm{~s}, 1 \mathrm{H}), 6.87$ - $8.03(\mathrm{~m}, 14 \mathrm{H}), 8.57(\mathrm{~s}, 1 \mathrm{H}) \mathrm{ppm} ; \mathrm{MS}, \mathrm{m} / \mathrm{z}(\%): 632(\mathrm{M}+, 2.9)$, 617 (M+-15, 100.0), 275 (21.8), 104 (59.2), 76 (44.3).

IR $(\mathrm{KBr}) \vee 1732(\mathrm{C}=\mathrm{O}) \mathrm{cm}^{-1} ;{ }^{1} \mathrm{H}$ NMR $\left(\mathrm{CDCl}_{3}\right) \delta 1.41(\mathrm{t}, 3 \mathrm{H}), 2.43(\mathrm{~s}, 3 \mathrm{H}), 2.66(\mathrm{t}, 2 \mathrm{H}), 3.79(\mathrm{~s}, 3 \mathrm{H}), 3.84(\mathrm{t}, 2 \mathrm{H}), 3.88(\mathrm{~s}, 3 \mathrm{H})$, $4.44(\mathrm{q}, 2 \mathrm{H}), 6.22(\mathrm{~s}, 1 \mathrm{H}), 6.69(\mathrm{~s}, 1 \mathrm{H}), 6.81(\mathrm{~s}, 1 \mathrm{H}), 7.30-7.48(\mathrm{~m}, 4 \mathrm{H}) \mathrm{ppm} ;{ }^{13} \mathrm{C} \mathrm{NMR}\left(\mathrm{CDCl}_{3}\right) \delta 14.18,21.14,26.92,45.84$, $55.81,56.43,62.19,85.56,108.46,110.49,122.59,125.39,130.06,131.78,136.88,138.71,143.14,147.37,149.93,150.56$, 157.96, $160.06 \mathrm{ppm} ; \mathrm{MS}, \mathrm{m} / \mathrm{z}(\%): 451(\mathrm{M}+, 8.9), 293$ (100.0), $91(18.3)$.

IR $(\mathrm{KBr}) v 1734(\mathrm{C}=\mathrm{O}) \mathrm{cm}^{-1} ;{ }^{1} \mathrm{H}$ NMR $\left(\mathrm{CDCl}_{3}\right) \delta 1.43(\mathrm{t}, 3 \mathrm{H}), 2.65(\mathrm{t}, 2 \mathrm{H}), 3.78(\mathrm{~s}, 3 \mathrm{H}), 3.84(\mathrm{t}, 2 \mathrm{H}), 3.88(\mathrm{~s}, 3 \mathrm{H}), 4.46(\mathrm{q}, 2 \mathrm{H})$, $6.20(\mathrm{~s}, 1 \mathrm{H}), 6.67(\mathrm{~s}, 1 \mathrm{H}), 6.78(\mathrm{~s}, 1 \mathrm{H}), 7.31-7.52(\mathrm{~m}, 4 \mathrm{H}) \mathrm{ppm} ; \mathrm{MS}, \mathrm{m} / z(\%): 473\left(\mathrm{M}^{+}+2,3.5\right), 471\left(\mathrm{M}^{+}, 10.1\right), 293(100.0), 91$ (18.9).

IR $(\mathrm{KBr}) v 1708(\mathrm{C}=\mathrm{O}) \mathrm{cm}^{-1} ;{ }^{1} \mathrm{H}$ NMR $\left(\mathrm{CDCl}_{3}\right) \delta 2.66(\mathrm{t}, 2 \mathrm{H}), 3.79(\mathrm{~s}, 3 \mathrm{H}), 3.84(\mathrm{t}, 2 \mathrm{H}), 3.88(\mathrm{~s}, 3 \mathrm{H}), 3.96(\mathrm{~s}, 3 \mathrm{H}), 6.23(\mathrm{~s}, 1 \mathrm{H})$ $6.69(\mathrm{~s}, 1 \mathrm{H}), 6.81(\mathrm{~s}, 1 \mathrm{H}), 7.31-7.48(\mathrm{~m}, 5 \mathrm{H}) \mathrm{ppm} ;{ }^{13} \mathrm{C} \mathrm{NMR}\left(\mathrm{CDCl}_{3}\right) \delta 27.00,45.79,52.86,55.83,56.45,85.82,108.41,110.66$, $121.58,125.01,130.22,131.90,136.85,138.88,142.83,147.58,149.67,150.70,158.01,160.64 \mathrm{ppm} ; \mathrm{MS}, \mathrm{m} / z(\%): 423\left(\mathrm{M}^{+}\right.$, 8.9), 293 (100.0), $91(21.4)$

IR $(\mathrm{KBr}) v 1705(\mathrm{C}=\mathrm{O}) \mathrm{cm}^{-1} ;{ }^{1} \mathrm{H}$ NMR $\left(\mathrm{CDCl}_{3}\right) \delta 2.43(\mathrm{~s}, 3 \mathrm{H}), 2.66(\mathrm{t}, 2 \mathrm{H}), 3.79(\mathrm{~s}, 3 \mathrm{H}), 3.84(\mathrm{t}, 2 \mathrm{H}), 3.88(\mathrm{~s}, 3 \mathrm{H}), 3.96(\mathrm{~s}, 3 \mathrm{H})$, $6.23(\mathrm{~s}, 1 \mathrm{H}), 6.69(\mathrm{~s}, 1 \mathrm{H}), 6.81(\mathrm{~s}, 1 \mathrm{H}), 7.31-7.48(\mathrm{~m}, 4 \mathrm{H}) \mathrm{ppm} ;{ }^{13} \mathrm{C} \mathrm{NMR}\left(\mathrm{CDCl}_{3}\right) \delta 21.15,26.92,45.78,52.86,55.82,56.44$, $85.68,108.47,110.51,122.54,125.36,130.10,131.79,136.83,138.78,142.83,147.39,149.86,150.61,157.92,160.51$ ppm; MS, $m / z(\%): 437\left(\mathrm{M}^{+}, 9.3\right), 293(100.0), 91(20.3)$.

IR $(\mathrm{KBr}) v 1704(\mathrm{C}=\mathrm{O}) \mathrm{cm}^{-1} ;{ }^{1} \mathrm{H}$ NMR $\left(\mathrm{CDCl}_{3}\right) \delta 2.62(\mathrm{t}, 2 \mathrm{H}), 3.75(\mathrm{~s}, 3 \mathrm{H}), 3.84(\mathrm{t}, 2 \mathrm{H}), 3.88(\mathrm{~s}, 3 \mathrm{H}), 3.95(\mathrm{~s}, 3 \mathrm{H}), 6.21(\mathrm{~s}, 1 \mathrm{H})$, $6.75(\mathrm{~s}, 1 \mathrm{H}), 7.01(\mathrm{~s}, 1 \mathrm{H}), 7.25-7.49(\mathrm{~m}, 4 \mathrm{H}) \mathrm{ppm} ; \mathrm{MS}, m / z(\%): 459\left(\mathrm{M}^{+}+2,4.1\right), 457\left(\mathrm{M}^{+}, 10.2\right), 293(100.0), 91(20.9)$.

${ }^{1} \mathrm{H} \mathrm{NMR}\left(\mathrm{CDCl}_{3}\right) \delta 2.90(\mathrm{t}, 2 \mathrm{H}), 3.86(\mathrm{~s}, 3 \mathrm{H}), 3.98(\mathrm{~s}, 3 \mathrm{H}), 4.09(\mathrm{t}, 2 \mathrm{H}), 6.62(\mathrm{~s}, 1 \mathrm{H}), 7.07-8.11(\mathrm{~m}, 11 \mathrm{H}) \mathrm{ppm} ;{ }^{13} \mathrm{C} \mathrm{NMR}$ $\left(\mathrm{CDCl}_{3}\right) \delta 26.16,47.94,56.01,56.16,86.68,107.02,111.55,116.32,123.02,125.85,127.32,127.64,128.66,129.68,141.00$ $141.42,142.82,144.47,148.48,151.12,161.81,168.40 \mathrm{ppm}$; MS, $m / z(\%): 486\left(\mathrm{M}^{+}, 8.2\right), 324(100.0)$

${ }^{1} \mathrm{H}$ NMR $\left(\mathrm{CDCl}_{3}\right) \delta 2.35(\mathrm{~s}, 3 \mathrm{H}), 2.89(\mathrm{t}, 2 \mathrm{H}), 3.88(\mathrm{~s}, 3 \mathrm{H}), 4.00(\mathrm{~s}, 3 \mathrm{H}), 4.08(\mathrm{t}, 2 \mathrm{H}), 6.57(\mathrm{~s}, 1 \mathrm{H}), 7.07-8.07(\mathrm{~m}, 10 \mathrm{H}) \mathrm{ppm} ;{ }^{13} \mathrm{C}$ NMR $\left(\mathrm{CDCl}_{3}\right) \delta 21.43,26.16,47.84,55.96,56.15,86.65,107.06,111.44,116.32,123.06,125.84,127.30,127.59,128.86$, $129.68,140.95,141.41,142.82,144.38,148.34,151.06,161.80,168.39$ ppm; MS, $m / z(\%): 500\left(\mathrm{M}^{+}, 6.7\right), 324(100.0)$.

${ }^{1} \mathrm{H}$ NMR $\left(\mathrm{CDCl}_{3}\right) \delta 2.91(\mathrm{t}, 2 \mathrm{H}), 3.86(\mathrm{~s}, 3 \mathrm{H}), 3.88(\mathrm{~s}, 3 \mathrm{H}), 4.01(\mathrm{t}, 2 \mathrm{H}), 6.59(\mathrm{~s}, 1 \mathrm{H}), 7.07-8.10(\mathrm{~m}, 10 \mathrm{H}) \mathrm{ppm} ; \mathrm{MS}, \mathrm{m} / z(\%)$ : $522\left(\mathrm{M}^{+}+2,3.9\right), 520\left(\mathrm{M}^{+}, 9.6\right), 324(100.0)$.

${ }^{1} \mathrm{H}$ NMR (DMSO-d $) \delta 2.82(\mathrm{t}, 2 \mathrm{H}), 3.75(\mathrm{~s}, 3 \mathrm{H}), 3.90(\mathrm{~s}, 3 \mathrm{H}), 4.12(\mathrm{t}, 2 \mathrm{H}), 6.75(\mathrm{~s}, 1 \mathrm{H}), 7.12-8.07(\mathrm{~m}, 9 \mathrm{H}) \mathrm{ppm} ;{ }^{13} \mathrm{C}$ NMR (DMSO-d $\left.\mathrm{d}_{6}\right) \delta 25.54,47.01,55.51,55.86,108.17,111.12,111.64,111.82,112.78,116.30,122.70,125.61,129.04,130.01,139.98$, 144.92, 146.08, 147.80, 150.67, 151.12, 161.21, 161.54 ppm; MS, $m / z(\%): 492\left(\mathrm{M}^{+}, 7.9\right), 324$ (100.0), 127 (22.1), 73 (25.0).

${ }^{1} \mathrm{H}$ NMR (DMSO-d 6 ) $\delta 2.82(\mathrm{t}, 2 \mathrm{H}), 3.75(\mathrm{~s}, 3 \mathrm{H}), 3.90(\mathrm{~s}, 3 \mathrm{H}), 4.13(\mathrm{t}, 2 \mathrm{H}), 6.68(\mathrm{~s}, 1 \mathrm{H}), 6.76-8.08(\mathrm{~m}, 9 \mathrm{H}) \mathrm{ppm} ;{ }^{13} \mathrm{C} \mathrm{NMR}$ (DMSO-d $\left.\mathrm{d}_{6}\right) \delta 25.54,46.92,55.51,55.86,108.19,111.12,111.63,111.84,112.78,116.19,122.70,125.67,129.05,130.01,140.01$, $144.96,146.07,147.80,150.68,151.06,157.46,161.10 \mathrm{ppm}$; MS, $m / z(\%): 476\left(\mathrm{M}^{+}, 10.6\right), 324(100.0)$.

IR $(\mathrm{KBr}) v 1647(\mathrm{C}=\mathrm{O}) \mathrm{cm}^{-1} ;{ }^{1} \mathrm{H}$ NMR (DMSO-d $\left.\mathrm{d}_{6}\right) \delta 2.40(\mathrm{~s}, 3 \mathrm{H}), 2.48(\mathrm{~s}, 3 \mathrm{H}), 2.71(\mathrm{t}, 2 \mathrm{H}), 3.79(\mathrm{~s}, 3 \mathrm{H}), 3.92(\mathrm{t}, 2 \mathrm{H}), 3.98(\mathrm{~s}$, $3 \mathrm{H}), 6.24(\mathrm{~s}, 1 \mathrm{H}), 6.82(\mathrm{~s}, 1 \mathrm{H}), 6.88(\mathrm{~s}, 1 \mathrm{H}), 7.42$ - $7.93(\mathrm{~m}, 4 \mathrm{H}) \mathrm{ppm}$; MS, $m / z(\%): 421\left(\mathrm{M}^{+}, 10.4\right), 293$ (100.0), 91 (19.1). $1 \mathrm{H}), 6.83(\mathrm{~s}, 1 \mathrm{H}), 6.88(\mathrm{~s}, 1 \mathrm{H}), 7.48-7.91(\mathrm{~m}, 4 \mathrm{H}) \mathrm{ppm}$; MS, $m / z(\%): 443\left(\mathrm{M}^{+}+2,5.1\right), 441\left(\mathrm{M}^{+}, 12.8\right), 293(100.0), 91(22.9)$. 
$\operatorname{IR}(\mathrm{KBr}) v 1616(\mathrm{C}=\mathrm{O}) \mathrm{cm}^{-1} ;{ }^{1} \mathrm{H}$ NMR $\left(\mathrm{CDCl}_{3}\right) \delta 2.47(\mathrm{~s}, 3 \mathrm{H}), 2.68(\mathrm{t}, 2 \mathrm{H}), 3.81(\mathrm{~s}, 3 \mathrm{H}), 3.87(\mathrm{t}, 2 \mathrm{H}), 3.90(\mathrm{~s}, 3 \mathrm{H}), 6.32(\mathrm{~s}, 1 \mathrm{H})$ $6.71(\mathrm{~s}, 1 \mathrm{H}), 6.84(\mathrm{~s}, 1 \mathrm{H}), 7.36-8.30(\mathrm{~m}, 9 \mathrm{H}) \mathrm{ppm} ;{ }^{13} \mathrm{C} \mathrm{NMR}\left(\mathrm{CDCl}_{3}\right) \delta 21.26,26.95,46.00,55.92,56.53,86.65,108.62,110.62$, $122.67,125.29,126.01,128.25,130.25,130.26,131.98,133.14,135.55,137.11,138.89,147.46,150.72,151.28,158.22,184.25$ ppm; MS, $m / z$ (\%): 483 (M+ 6.7$), 293$ (100.0), 91 (16.8), 105 (66.8).

24d

$24 \mathrm{e}$

$24 \mathrm{f}$

$24 \mathrm{~g}$
IR $(\mathrm{KBr}) v 1616(\mathrm{C}=\mathrm{O}) \mathrm{cm}^{-1} ;{ }^{1} \mathrm{H}$ NMR $\left(\mathrm{CDCl}_{3}\right) \delta 2.67(\mathrm{t}, 2 \mathrm{H}), 3.79(\mathrm{~s}, 3 \mathrm{H}), 3.87(\mathrm{t}, 2 \mathrm{H}), 3.91(\mathrm{~s}, 3 \mathrm{H}), 6.34(\mathrm{~s}, 1 \mathrm{H}), 6.70(\mathrm{~s}, 1 \mathrm{H})$, $6.84(\mathrm{~s}, 1 \mathrm{H}), 7.35-8.32(\mathrm{~m}, 9 \mathrm{H}) \mathrm{ppm}$; MS, $m / z(\%): 505\left(\mathrm{M}^{+}+2,3.9\right), 503\left(\mathrm{M}^{+}, 9.5\right), 293(100.0), 91(17.2), 105(67.8)$.

IR $(\mathrm{KBr}) v 1672(\mathrm{C}=\mathrm{O}), 3385(\mathrm{NH}) \mathrm{cm}^{-1} ;{ }^{1} \mathrm{H}$ NMR $\left(\mathrm{CDCl}_{3}\right) \delta 2.68(\mathrm{t}, 2 \mathrm{H}), 3.81(\mathrm{~s}, 3 \mathrm{H}), 3.83(\mathrm{t}, 2 \mathrm{H}), 3.91(\mathrm{~s}, 3 \mathrm{H}), 6.29(\mathrm{~s}, 1 \mathrm{H})$, 6.63 - $8.70(\mathrm{~m}, 12 \mathrm{H}), 11.75(\mathrm{~s}, 1 \mathrm{H}) \mathrm{ppm}$; MS, $\mathrm{m} / \mathrm{z}(\%): 484\left(\mathrm{M}^{+}, 7.6\right), 293(100.0)$.

IR (KBr) $v 1670(\mathrm{C}=\mathrm{O}), 3386(\mathrm{NH}) \mathrm{cm}^{-1} ;{ }^{1} \mathrm{H}$ NMR $\left(\mathrm{CDCl}_{3}\right) \delta 2.47(\mathrm{~s}, 3 \mathrm{H}), 2.68(\mathrm{t}, 2 \mathrm{H}), 3.80(\mathrm{~s}, 3 \mathrm{H}), 3.85(\mathrm{t}, 2 \mathrm{H}), 3.89(\mathrm{~s}, 3 \mathrm{H})$, $6.22(\mathrm{~s}, 1 \mathrm{H}), 6.66-8.65(\mathrm{~m}, 11 \mathrm{H}), 11.73(\mathrm{~s}, 1 \mathrm{H}) \mathrm{ppm}$; MS, $m / z(\%)$ : $498\left(\mathrm{M}^{+}, 9.5\right), 293(100.0)$.

IR $(\mathrm{KBr}) v 1670(\mathrm{C}=\mathrm{O}), 3388(\mathrm{NH}) \mathrm{cm}^{-1} ;{ }^{1} \mathrm{H} \mathrm{NMR}\left(\mathrm{CDCl}_{3}\right) \delta 2.66(\mathrm{t}, 2 \mathrm{H}), 3.81(\mathrm{~s}, 3 \mathrm{H}), 3.83(\mathrm{t}, 2 \mathrm{H}), 3.89(\mathrm{~s}, 3 \mathrm{H}), 6.32(\mathrm{~s}, 1 \mathrm{H})$, $6.66-8.66(\mathrm{~m}, 11 \mathrm{H}), 11.74(\mathrm{~s}, 1 \mathrm{H}) \mathrm{ppm} ; \mathrm{MS}, \mathrm{m} / \mathrm{z}(\%): 520\left(\mathrm{M}^{+}+2,2.7\right), 518\left(\mathrm{M}^{+}, 8.3\right), 293(100.0)$.

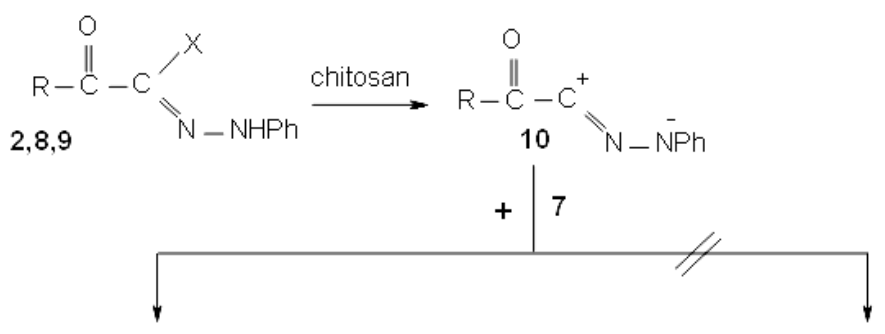<smiles>[R]C(=O)c1nn(-c2ccccc2)cc1C(=O)C1=NN([Al])C2(C)C(=O)N1C[C@H](I)c1cc(OC)c(OC)cc12</smiles>

\section{$\mathrm{R} / \mathrm{Ar}$}

a: $\mathrm{CH}_{3} / \mathrm{C}_{6} \mathrm{H}_{5}$

b: $\mathrm{CH}_{3} / 4-\mathrm{CH}_{3} \mathrm{C}_{6} \mathrm{H}_{4}$

c: $\mathrm{CH}_{3} / 4-\mathrm{ClC}_{6} \mathrm{H}_{4}$

d: $\mathrm{C}_{2} \mathrm{H}_{5} \mathrm{O} / \mathrm{C}_{6} \mathrm{H}_{5}$

e: $\mathrm{C}_{2} \mathrm{H}_{5} \mathrm{O} / 4-\mathrm{CH}_{3} \mathrm{C}_{6} \mathrm{H}_{4}$

Figure 3. Synthesis of pyrazoles 13.

other hand, $H(5)$ is linked to the carbon attached to nitrogen atom and thus it's deshielded to appear in the region $\delta 7.5-8.5 \mathrm{ppm}[26-28]$. The ${ }^{1} \mathrm{H}$ NMR spectra of isolated reaction products revealed, in each case, a singlet signal at $\delta 8.5 \mathrm{ppm}$ which indicates the presence of the pyrazole $\mathrm{H} \mathrm{(5)} \mathrm{rather} \mathrm{than} \mathrm{H}(4)$ in the structure of

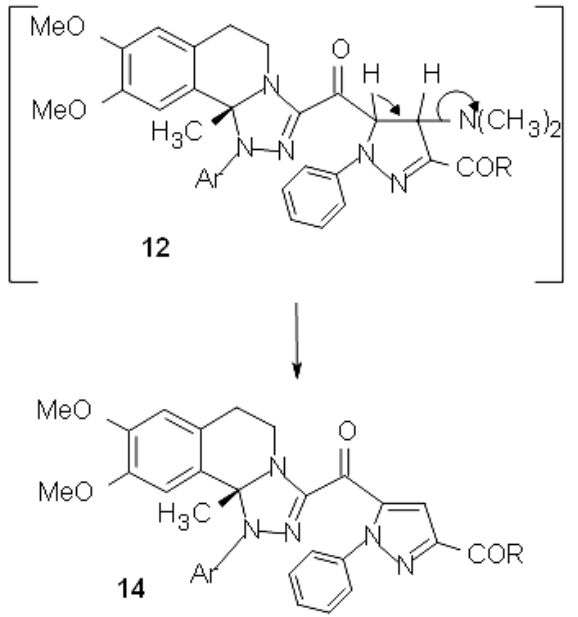

$\mathrm{R} / \mathrm{Ar}$

f: $\mathrm{C}_{2} \mathrm{H}_{5} \mathrm{O} / 4-\mathrm{ClC}_{6} \mathrm{H}_{4}$

g: $\mathrm{C}_{6} \mathrm{H}_{5} / \mathrm{C}_{6} \mathrm{H}_{5}$

h: $\mathrm{C}_{6} \mathrm{H}_{5} / 4-\mathrm{CH}_{3} \mathrm{C}_{6} \mathrm{H}_{4}$

i: $\mathrm{C}_{6} \mathrm{H}_{5} / 4-\mathrm{ClC}_{6} \mathrm{H}_{4}$

the isolated products.

The proposed mechanism leading to the formation of the latter product suggested that the studied reaction starts with regioselective 1,3-dipolar cycloaddition of nitrilimines 10 to $\mathrm{C}=\mathrm{C}$ of the enaminones 7 to afford the cycloadducts 11 which gave the pyrazole derivatives 13 
via elimination of dimethylamine and the other isomer 14 resulting from 12 was discarded (Figure 3).

Treatment of thioanilide [9] 15a with hydrazonoyl halides 16 in refluxing ethanol in the presence of chitosan under microwave irradiation for $10 \mathrm{~min}$ yielded only one isolable product 20 as indicated by TLC and ${ }^{1} \mathrm{H}$ NMR of the crude reaction product (Figure 4).

The structure of the products was inferred from their elemental analyses, spectral data and by their alternative synthesis. Thus reaction of $15 \mathrm{~b}$ with 16 gave products identical in all respects (mp, IR, ${ }^{1} \mathrm{H}$ NMR, MS) with products 20 which formed by the reaction of 15 a with 16 , respectively (Figure 4). For example, the ${ }^{1} \mathrm{H}$ NMR of $20 \mathrm{a}$ showed triplet and quartet signals at $\delta 1.41$ and at $\delta$ $4.44 \mathrm{ppm}$ respectively, assignable to the ethoxycarbonyl group, and a singlet signal at $\delta 6.81 \mathrm{ppm}$ assignable to methylidene proton in addition to the signals of the isoquinoline moiety. Its IR spectrum showed the characteristic ester carbonyl absorption band at $1732 \mathrm{~cm}^{-1}$.

Two possible structures 20 and 21 can be suggested for the products resulting from the reaction of 15 with hydrazonoyl halides 16 or nitrilimine 17 . Structure 21 was ruled out because the reaction product was recov- ered unchanged after treatment with mercuric oxide in boiling acetic acid.

To account for the formation of 20, two possible pathways are proposed. In the first way (path A), the reaction led to the formation of thiohydrazones 18 followed by elimination of arylamine to give 20 . The second path (path B), nitrilimines 17 cycloadded to the $\mathrm{C}=$ $\mathrm{S}$ double bond to give the intermediate 19 which upon elimination of arylamine led to 20 .

To study the effect of the carbonyl group on the reactivity of the hydrazonoyl halides, we investigated the reaction of $\alpha$-ketohydrazonoyl halides 2, 9, 22 with thioanilides 15 . Thus treatment of $15 \mathrm{a}$ or $15 \mathrm{~b}$ with hydrazonoyl halides 2, 9, 22 in refluxing ethanol in the presence of chitosan under microwave irradiation for 10 min gave the corresponding thiadiazole derivatives 24 (Figure 5).

The structures of the products 24 were supported by their elemental analyses and spectral data. The other possible structures 25 and 26 were excluded on the basis of elemental analysis and spectral data. For example, their IR spectra lacked the carbonyl absorption band while such band is present in the spectra of the product

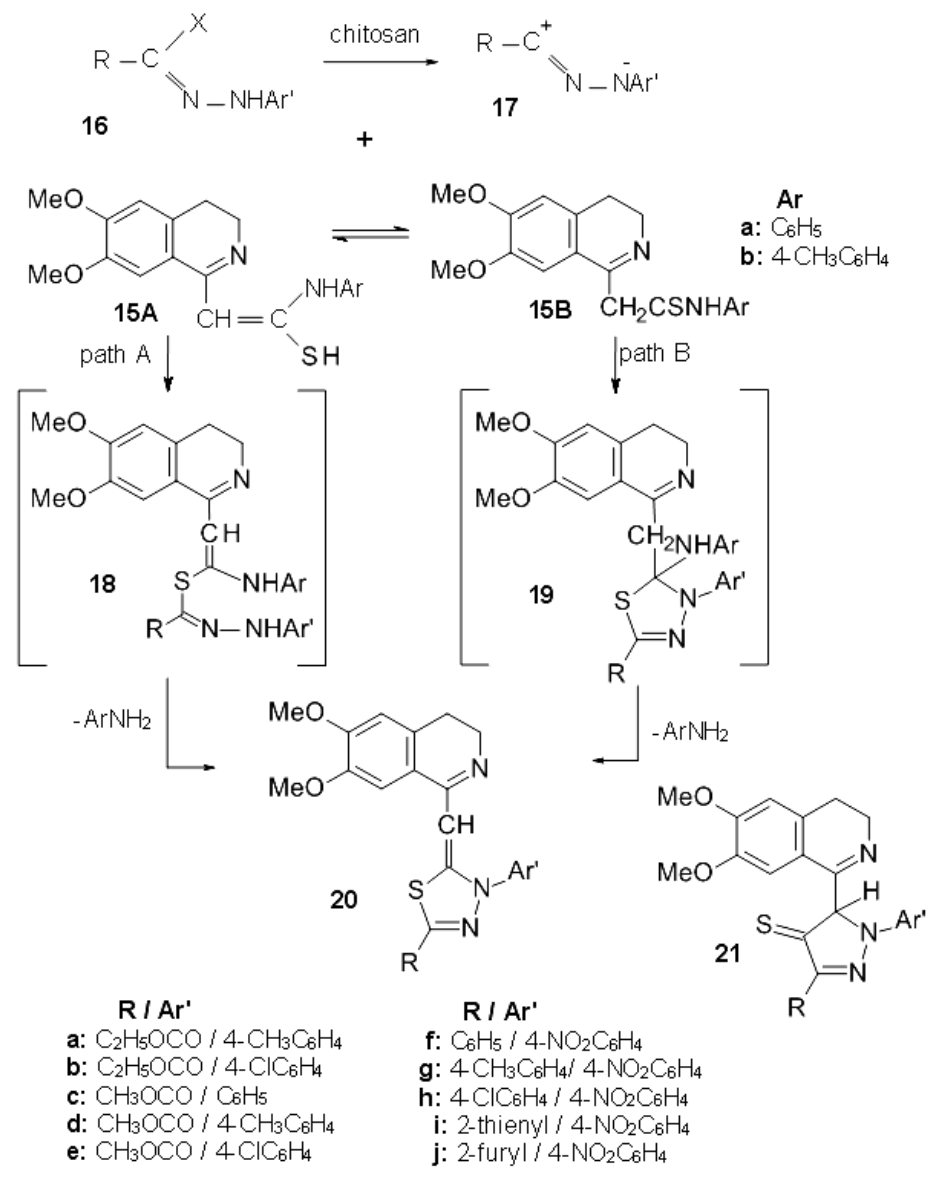

Figure 4. Synthesis of $[1,3,4]$ thiadiazolyl isoquinolines 20 . 


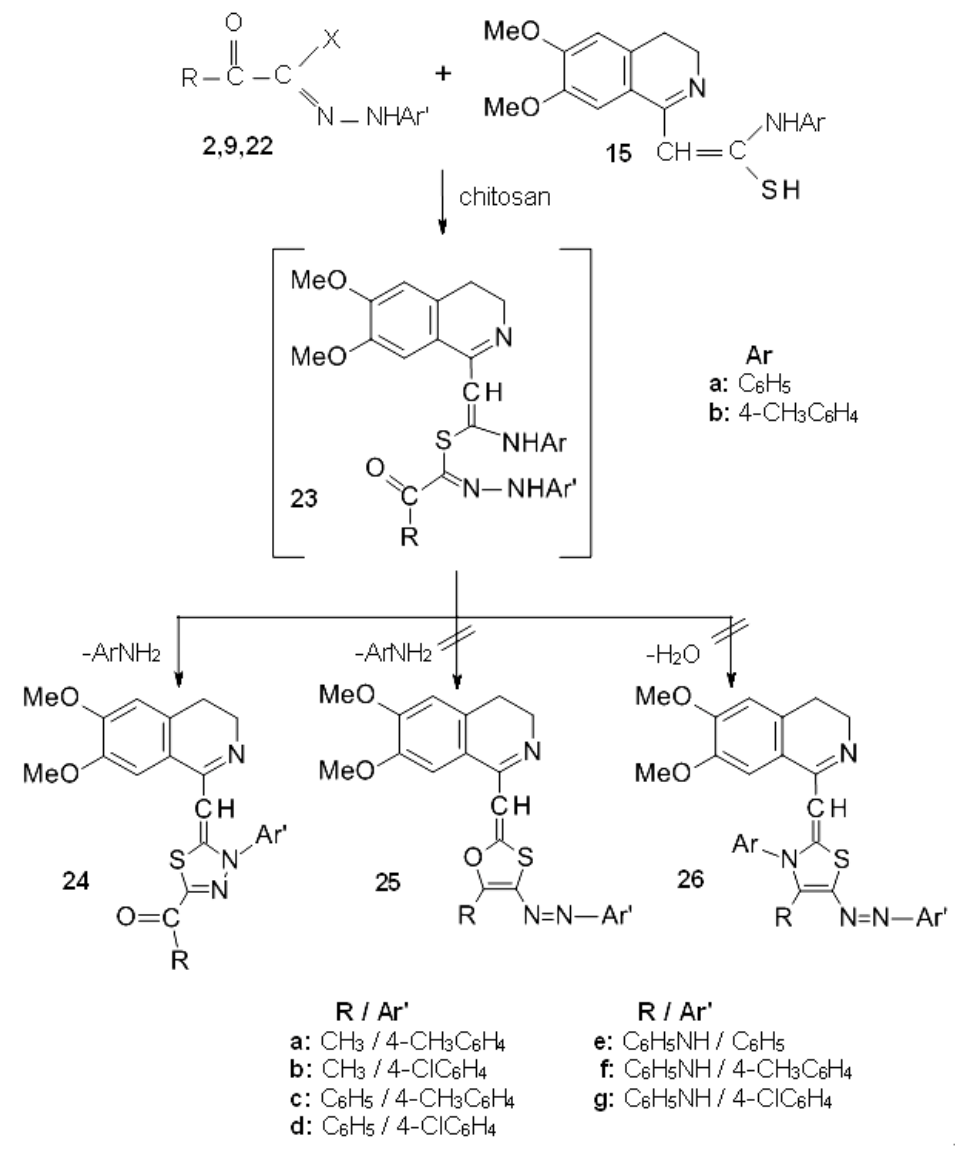

Figure 5. Synthesis of $[1,3,4]$ thiadiazolyl isoquinolines 24 .

24. The structure of the latter products proved that the carbonyl group has no effect on the course of this reaction.

\section{REFERENCES}

[1] Geigy, J.R. and S-Triazolo A.G. (1968) [3,4-a] Isoquinolines, Neth Apple. Chemical Abstract, 68, 69003.

[2] Solecka, J., Rajnisz, A. and Laudy, A.E. (2009) A novel isoquinoline alkaloid, DD-carboxypeptidase inhibitor, with antibacterial activity isolated from Streptomyces $\mathrm{sp}$. 8812. Part I: Taxonomy, fermentation, isolation and biological activities. Journal Antibiotic, 6, 575-580. doi:10.1038/ja.2009.85

[3] Maryanoff, B.E., McComsey, D.F., Costanzo, M.J., Setler, P.E., Gardocki, J.F., Shank, R.P. and Schneider, C.R. (1984) Pyrroloisoquinoline antidepressants. Potent, enantioselective inhibition of tetrabenazine-induced ptosis and neuronal uptake of norepinephrine, dopamine, and serotonin. Journal Medicine Chemical, 27, 943-946. doi:10.1021/jm00374a001

[4] Tiwaria, R.K., Singha, D., Singha, J., Chhillarb, A.K., Chandraa, R. and Vermaa, A.K., (2006) Synthesis, antibacterial activity and QSAR studies of 1, 2-disubstituted6,7-dmethoxy-1, 2, 3, 4-tetrahydroisoquinolines. Europe Journal Medicine Chemical, 41, 40-49. doi:10.1016/j.ejmech.2005.10.010
[5] Bentley, K.W. (2003) $\beta$-Phenylethylamines and the isoquinoline alkaloids. Natural Product Reports, 20, 342365. doi:10.1039/b111626f

[6] Fülöp, F., Lazar, L., El-Gharib, M.S.A. and Bernáth, G. (1990) Saturated heterocycles. Part 166. Synthesis of 1, 5, 6, 10b-tetrahydro-1, 2, 4-triazolo [3,4-a] isoquinoline. Pharmazie, 45, 60-61.

[7] Ito, S., Kakehi, A., Matsuno, T. and Yoshida, J. (1980) The preparation of 3-phenyl [1,2,4] triazolo [4,3-a] pyridines and their Benzologs from N-(phenylsulfonyl) benzohydazonoyl chloride and pyridines. Bull Chemical Socience Japenese, 53, 2007-2011. doi:10.1246/bcsj.53.2007

[8] Hassaneen, H.M., Abdallah, T.A. and Awad, E. (2009) A facile access for synthesis of novel isoquiunoline-based heterocycles. Heterocycles, 78, 1507-1522. doi:10.3987/COM-09-11648

[9] Abdallah, T.A., Abdelhadi, H.A. and Hassaneen, H.M. (2002) Reactivity of 1-Methylisoquinoline. Synthesis of 2-(1-Isoquinolinemethylidene)-1, 3, 4-Thiadiazole Derivatives. Phosphorus Sulfur and Silicon, 177, 59-66. doi:10.1080/10426500210218

[10] Elwan, N.M., Abdelhadi, H.A. and Hassaneen, H.M. (1996) Synthesis of $[1,2,4]$ triazolo $[3,4-a]$ isoquinolines and pyrrolo $[2,1-a]$ Isoquinolines using $\alpha$-keto hydrazonoyl halides. Tetrahedron, 52, 3451-3456. doi:10.1016/0040-4020(96)00024-5 
[11] Abdallah, T.A., Hassaneen, H.M. and Abdelhadi, H.A. (2009) Synthesis of tetra- and penta- heterocyclic compounds incorporated isoquinoline moiety, Heterocycles, 78, 373-378. doi:10.3987/COM-08-11481

[12] Awad, E.M., Elwan, N.M., Hassaneen, H.M., Linden A. and Heimgartner, H. (2002) New routes to fused isoquinoline, Helvetica Chimica Acta, 85, 320-331. doi:10.1002/1522-2675(200201)85:1<320::AID-HLCA3 20>3.0.CO;2-X

[13] Al-matar, H.M., Khalil, K.D., Meier, H. and Elnagdi, M.H. (2008) Chitosan as heterogeneous catalyst in Michael additions: The reaction of cinnamonitriles with active methyls, active methylenes and phenols, Arkivoc Xvi, 288-301.

[14] Guibal, E. (2005) Heterogeneous catalysis on chitosan-based materials: a review, Progress in Polymer Science, 30, 71-109. doi:10.1016/j.progpolymsci.2004.12.001

[15] Bollini, M., Gonzalez, M. and Bruno, A. (2009) Microwave-assisted rapid and efficient synthesis of C-alkyl imidazoisoquinolinone derivatives, Tetrahedron Letters, 50, 1507-1509. doi:10.1016/j.tetlet.2009.01.083

[16] Andrade, C.K.Z., Barreto, A.S. and Silva, W.A. (2008) Microwave assisted solvent-, support- and catalyst-free synthesis of enaminones, Arkivoc Xii, 226-232.

[17] Lidstrom, P.J., Tierney, J., Wathey, B. and Westman, J. (2001) Microwave assisted organic synthesis - a review, Tetrahedron, 57, 9225-9283. doi:10.1016/S0040-4020(01)00906-1

[18] Bortolini, O., D’Agostino, M., De Nino, A., Maiuolo, L., Nardi, M. and Sindona, G. (2008) Solvent-free, microwave assisted 1,3-cycloaddition of nitrones with vinyl nucleobases for the synthesis of N,O-nucleosides, Tetrahedron, 64, 8078-8081.doi:10.1016/j.tet.2008.06.074

[19] El Ashry, E.H. and Kassem, A.A. (2006) Account of microwave irradiation for accelerating organic reactions, Arkivoc Xii, 1-16.

[20] Nair, M.D. and Metha, S.R. (1967) Long range coupling in heterocyclic compounds, Indian Journal Chemical, 12B, 5 .
[21] Battersby, A.R., Openshaw, H.T. and Wood, H.C.S. (1953) The synthesis of emetine and related compounds. Part II. The synthesis of $( \pm$ )-rubremetinium bromide, Journal Chemical Socience, 2463-2470.

[22] Gomha, S.M. and Riyadh, S.M. (2009) Synthesis of triazolo $[4,3-b][1,2,4,5]$ tetrazines and triazolo $[3,4-b][1,3,4]$ thiadiazines using chitosan as ecofriendly catalyst under microwave irradiation, Arkivoc Xii, 58-68.

[23] Hassaneen, H.M., Hassaneen, H.M.E. and Mohammed, Y.Sh. (2011) Synthesis, Reactions and Antibacterial Activity of 3-Acetyl $[1,2,4]$ triazolo $[3,4-a]$ isoquinoline Derivatives using Chitosan as Heterogeneous Catalyst under Microwave Irradiation, Verlag der Zeitschrift für Naturforschung, 66b, 299-310.

[24] Dawood, K. M. (2005) Synthesis of Spiro-pyrazole-3, 3'thiopyrano $[2,3-b]$ pyridines and Azolo [a] pyrido $\left[2^{\prime}, 3^{\prime}: 5,6\right]$ thiopyrano $[3,4-d]$ pyrimidines as New Ring Systems with Antifungal and Antibacterial Activities, Journal Heterocyclic Chemical, 42, 221-225. doi:10.1002/jhet.5570420207

[25] Farag, A.M., Mayhoubb, A.S., Barakatb, S.E. and Bayomi, A.H. (2008) Regioselective synthesis and antitumor screening of some novel N-phenylpyrazole derivatives. Bioorganic and Medicinal Chemistry, 16, 881-889. doi:10.1016/j.bmc.2007.10.015

[26] Komarova, E.S., Makarov, V.A., Alekseeva, G.V. and Granik, V.G. (2006) Synthesis of derivatives of a new heterocyclic system pyrazolo $[3,4-b]$ pyrido $[1 ', 2$ ': 1,2$]$ imidazo [4,5-d] pyridine, Russian Chemical Bull International Edition, 55, 735-740. doi:10.1007/s11172-006-0322-Z

[27] He, F.Q., Liu, X.H., Wang, B.L. and Li, Z.M. (2008) Synthesis and biological activities of novel bis-heterocyclic pyrrodiazole derivatives, Heteroatom Chemical, 19, 21-27. doi:10.1002/hc.20369

[28] Amer, F.A., Hammouda, M., El-Ahl, A.S. and Abdelwahab, B.F. (2007) Synthesis of Important New Pyrrolo $[3,4-c]$ pyrazoles and Pyrazolyl-Pyrrolines from Heterocyclic $\beta$-Ketonitriles, Journal Chinese Chemical Socience, 54, 1543-1552. 